\title{
Heavy Ion Beams for Radiobiology: Dosimetry and Nanodosimetry at HIL
}

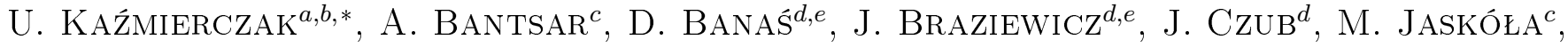

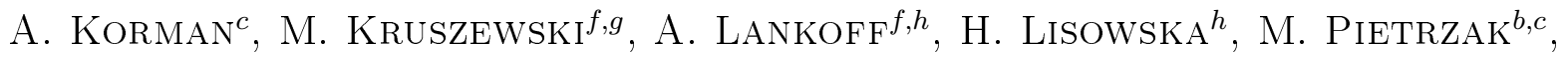 \\ S. Pszona ${ }^{c}$, T. StęPKOWski ${ }^{f}$, Z. Szefliński ${ }^{a}$ AND M. WojeWÓDzKA ${ }^{f}$ \\ ${ }^{a}$ Heavy Ion Laboratory, University of Warsaw, L. Pasteura 5A, 02-093 Warsaw, Poland \\ ${ }^{b}$ Faculty of Physics, University of Warsaw, L. Pasteura 5, 02-093 Warsaw, Poland \\ ${ }^{c}$ National Centre for Nuclear Research, A. Sołtana 7, 05-400 Otwock-Świerk, Poland \\ ${ }^{d}$ Institute of Physics, Jan Kochanowski University, Świętokrzyska 15, 25-406 Kielce, Poland \\ ${ }^{e}$ Holycross Cancer Center, Artwińskiego 3, 25-734 Kielce, Poland \\ ${ }^{f}$ Institute of Nuclear Chemistry and Technology, Dorodna 16, 03-195 Warsaw, Poland \\ ${ }^{g}$ Institute of Rural Health, K. Jaczewskiego 2, 20-090 Lublin, Poland \\ ${ }^{h}$ Institute of Biology, Jan Kochanowski University, Świętokrzyska 15, 25-406 Kielce, Poland
}

\begin{abstract}
Ionizing radiation induces a variety of DNA lesions, including single and double strand breaks. Large energy deposition precisely localized along the ion track that occurs in the case of heavy ion irradiation can lead to complex types of DNA double strand breaks in exposed biological material. The formation of nuclear double strand breaks triggers phosphorylation of histone $\mathrm{H} 2 \mathrm{AX}$, which can be microscopically visualized as foci in the $\gamma$-H2AX assay. Studies with a carbon ion beam are being carried out at the Heavy Ion Laboratory of the University of Warsaw. The $\gamma$-H2AX assay as a method of measuring the biological response of cells irradiated with ${ }^{12} \mathrm{C}$ ions as well as the frequency cluster size distributions obtained in the nanodosimetry experiment at HIL will be presented.
\end{abstract}

DOI: $10.12693 /$ APhysPolA.127.1516

PACS: 87.53.Bn, 87.53.-j

\section{Introduction}

Research in the field of the biological effect induced by heavy charged particles is needed for radiotherapy and space radiation protection (as reviewed by Durante [1]). The main reason for using protons and heavier ions in tumour therapy is their physical depth-dose distribution in tissue, characterized by a small dose at the entrance and a distinct maximum (called the Bragg peak) near the end of the range. Carbon beams, used in hadron therapy, have the additional advantage of a markedly increased biological effectiveness characterized by enhanced ionization density in the individual tracks of the heavy particles, where DNA damage becomes clustered and therefore more difficult to repair [2].

In response to radiation-induced double strand breaks (DSBs) histone $\mathrm{H} 2 \mathrm{AX}$ phosphorylation is induced and the concentration of DNA repair proteins to the damaged chromatin starts. The $\gamma-\mathrm{H} 2 \mathrm{AX}$ assay allows for visualization of formed foci and therefore was used as a method of measuring the biological response of cells irradiated with ${ }^{12} \mathrm{C}$ ions at the Heavy Ion Laboratory of the University of Warsaw (HIL). The number of foci can be an indicator of the deposited dose.

\footnotetext{
* corresponding author; e-mail: urszula.kazmierczak@fuw.edu.pl
}

The probability of DSB forming by carbon ions has been experimentally simulated by nanodosimetry method based on use of the Jet Counter. The ionization cluster size distribution, ICSD, created by $70 \mathrm{MeV}$ carbon ions at nanometre size comparable to a short segment of DNA molecule was measured. The first moment of ICSD as well as the cumulative probability distribution for cluster sizes of 2 and higher were determined. These parameters are the candidates for the new descriptors of radiation damage at DNA level [3].

\section{Materials and methods of irradiation}

\subsection{Irradiation facility}

The experimental setup has been described previously $[4,5]$ and therefore only a short review is presented here. The HIL cyclotron allows for acceleration of almost all types of ions which can potentially be used in heavy-ion tumour therapy. The accessible setup provides the possibility to irradiate biological samples under physiological conditions by ions at energies corresponding to the Bragg peak. In described investigations a ${ }^{12} \mathrm{C}$ ion beam was used. A schema of the facility for radiobiological (in a straight line) and nanodosimetry (jet counter at an angle of $45^{\circ}$ ) studies is shown in Fig. 1 .

\subsubsection{Setup for nanodosimetry}

The nanodosimetric measurements were performed at the jet counter facility using the single-ion-counting method. The ICSD spectra were measured based on 


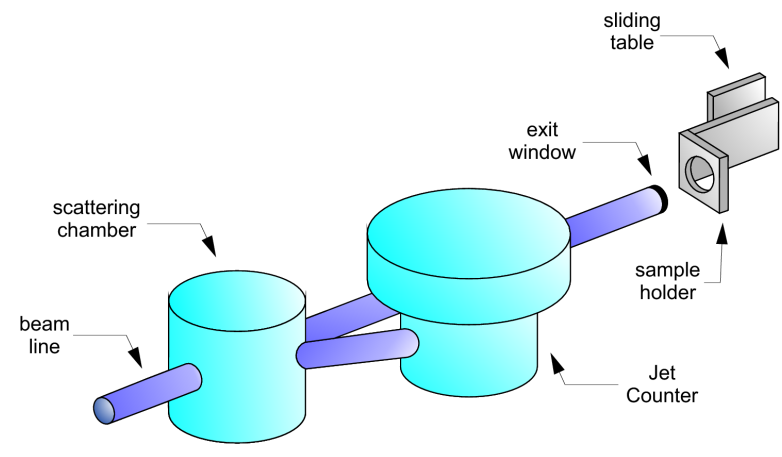

Fig. 1. Schematic view of the setup with the horizontal beam line for radiobiological and nanodosimetry studies at HIL.

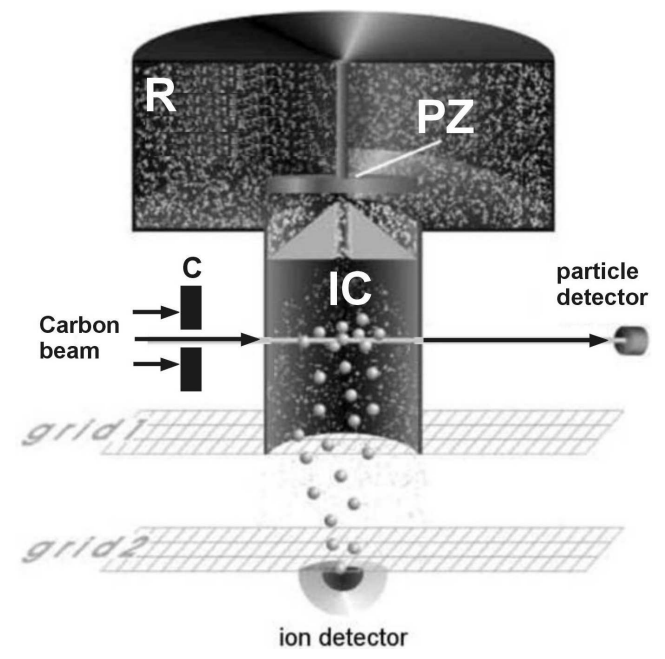

Fig. 2. Jet counter - schematic view. IC - interaction chamber, $\mathrm{R}$ - gas reservoir, $\mathrm{PZ}$ - piezoelectric valve, $\mathrm{C}$ - collimator.

counting the number of ionizations in coincidence with the ionizing particle (carbon ion) after passing through the simulated nanometric volume. A schematic view of the jet counter device is presented in Fig. 2.

A simulated nanometre-sized site (SNS) is obtained in the device by pulse expansion of nitrogen (which leads to a nitrogen jet) to the volume of the interaction chamber (IC). By analogy with experimental microdosimetry, it is assumed here that the interaction of ionizing particles in nanometre-sized biological structures can also be studied in gaseous volumes if the volume sizes, expressed in mass per area, are comparable to those of the real radiation-sensitive biological targets.

The volume of the IC is of cylindrical shape, $10 \mathrm{~mm}$ in diameter and $10 \mathrm{~mm}$ in height, walled by $1 \mathrm{mg} / \mathrm{cm}^{2}$ mylar. The nitrogen jet is created by the pulse-operated valve $(\mathrm{PZ})$ which injects nitrogen from a gas reservoir $(\mathrm{R})$ through a nozzle with an orifice $1 \mathrm{~mm}$ in diameter to the IC. The nitrogen gas density (and, in particular, its maximum which defines the SNS) was determined and controlled in an electron-transmission experiment, using electrons with known energy and known total scattering cross-section (for details see Bantsar [6]). Carbon ions are focused by a movable collimator (C) of $3 \mathrm{~mm}$ height and $1 \mathrm{~mm}$ width that can move along the axis of the carbon beam by a distance of up to $5 \mathrm{~mm}$ from the centre of the cylinder of the IC. Nitrogen molecules in the gas jet are ionized by single carbon ions passing the sensitive volume by forming ionization clusters of different sizes ICSD. All ions created after ionization are extracted by an electric field created by grids 1 and 2, and guided to an ion detector for counting. Nanodosimetry of $76 \mathrm{MeV}$ carbon ions, approximately of the same energy as used for radiobiological experiment, was performed. The results in form of ICSD spectrum are discussed in Sect. 3.2.

\subsubsection{Setup for radiobiological studies}

At the setup with a horizontal beam line for radiobiological studies an independent Si-detector system is used for beam diagnosis and monitoring. One of the detectors is applied to monitor particle flux by the intensity of the scattered particles and a second is used to measure the particle flux at the position of the cell container [4].

In order to obtain a homogeneous radiation field over the area of $1 \times 1 \mathrm{~cm}^{2}$ of the exit window, the ion beam is passively spread out by a gold foil. The first silicon detector is placed in the scattering chamber at an angle of $20^{\circ}$. It ensures on-line beam monitoring during the exposure in addition to counting individual particles for dosimetric purposes. The beam passes through a $1.8 \mathrm{~m}$ horizontal beam line and lets into the air by a $2.3 \mathrm{mg} / \mathrm{cm}^{2}$ havar exit window. Ions pass through an air layer and reach a Petri dish with the biological sample or alternatively the second detector. This is a surface barrier detector fastened outside the exit window to an $x-y-z$ stepping motor that moves across a two-dimensional array in $1 \mathrm{~mm}$ steps on the $1 \times 1 \mathrm{~cm}^{2}$ area of the beam to determine the distribution of the scattered ion intensity. The beam dosimetry is based on single-particle counting. The measured flux of the ions registered by the $0^{\circ}$ detector in the air, outside the exit window, is proportional to the count rate of the $20^{\circ}$ detector registering scattered ions in the chamber. The measured relation of the number of ions reaching the detector at an angle of $20^{\circ}$ to the number of ions registered at $0^{\circ}$, taking into account the type of ions and their energy, is used to calculate the total fluence of carbon ions as well as the dose deposited in the biological material [7].

\section{2. $\gamma$-H2AX foci as a marker of DNA double strand breaks}

The homogeneity of the ion beam and the number of ions hitting the cell container were verified experimentally by the $\gamma-\mathrm{H} 2 \mathrm{AX}$ assay. It is well-known that irradiation of cells with ${ }^{12} \mathrm{C}$ ions, characterized by extremely localized energy deposition, leads to complex types of DNA double strand breaks in the exposed material. These breaks represent a severe form of DNA damage because they result in chromosomal aberration [8] and are powerful inducers of cell death by apoptosis [9]. 
DSBs are induced linearly with radiation dose and apart from ionizing radiation only a few other environmentally relevant agents or processes are known to generate significant levels of them [10]. Double strand breaks within seconds to minutes following exposure to ionizing radiation activate a signalling pathway, marked by phosphorylation of histone 2AX (H2AX) [11]. Phosphorylated $\mathrm{H} 2 \mathrm{AX}$ (designated as $\gamma-\mathrm{H} 2 \mathrm{AX}$ ) is rapidly accumulated at sites of DSBs [12] and, therefore, can be microscopically visualized as foci by immunocytochemistry [13]. Phosphorylation of $\mathrm{H} 2 \mathrm{AX}$ provides an ideal functional marker to measure the induction of DSBs caused by radiation [14]. On the other hand, this marker can be used as an indicator of the dose.

\subsubsection{Cell line and culture conditions}

Chinese hamster ovary cells (CHO-K1) have been used as a suitable biological system for our studies. The selected cell line has an adhesion ability, which is important in view of the irradiation, when the dish with the cells is vertically mounted. The cells were incubated in a humidified atmosphere at $37^{\circ} \mathrm{C}$ with $5 \% \mathrm{CO}_{2}$. Biological samples were cultured in a medium containing $10 \%$ fetal bovine serum (FBS), 1\% penicillin and streptomycin.

Prepared samples were exposed to ionising radiation of high linear energy transfer (LET) ${ }^{12} \mathrm{C}$ ions. Irradiated cells were placed back in the incubator for $30 \mathrm{~min}$. Then samples were fixed in $2 \%$ formaldehyde and processed for the $\gamma$-H2AX assay. Next, the cells were mounted with Vectashield mounting medium for fluorescence with DAPI (Vector Laboratories, Burlingame, CA, USA). The number of ion tracks registered in individual cells was counted under a NIKON TI Eclipse A1 confocal microscope.

\section{Results}

\subsection{Radiobiological studies}

Representative images of cells processed for the $\gamma$ H2AX assay are shown in Fig. 3. Part (a) of the figure presents a non-irradiated cell with a spontaneous focus. It is a single focus located in the centre of the nucleus.

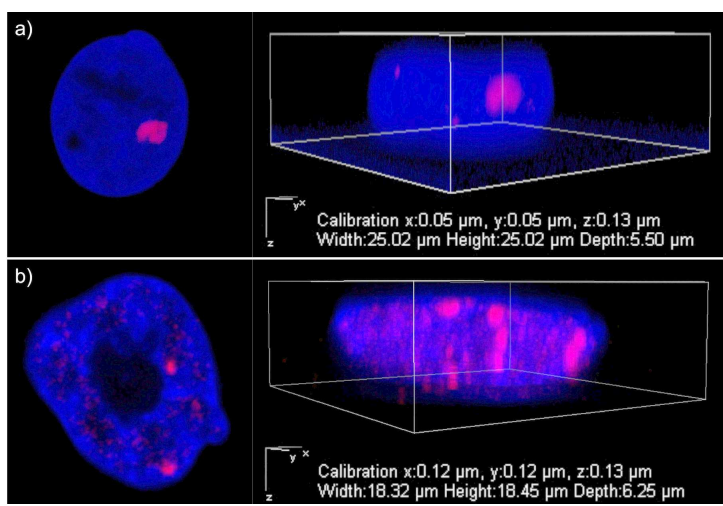

Fig. 3. CHO-K1 cell nuclei processed for the $\gamma$-H2AX assay: (a) control cell and (b) irradiated cell. Both cells are viewed from above and in cross-section.
In contrast with the control cell, in Fig. 3b a cell irradiated with $70 \mathrm{MeV}{ }^{12} \mathrm{C}$ ions is shown. Foci patterns extending across the entire nucleus, along the tracks of carbon ions, are clearly visible. The arrangement of the foci pattern maps the traces of three carbon ions that passed through the cell nucleus.

\subsection{Nanodosimetry of carbon beam}

The ionization cluster size distributions ICSD obtained in the experiment with $76 \mathrm{MeV}$ carbon ions that passed the centre of the IC in molecular nitrogen with areal density $\mathrm{D} \rho=0.32 \mu \mathrm{g} / \mathrm{cm}^{2}$ are shown in Fig. 4 .

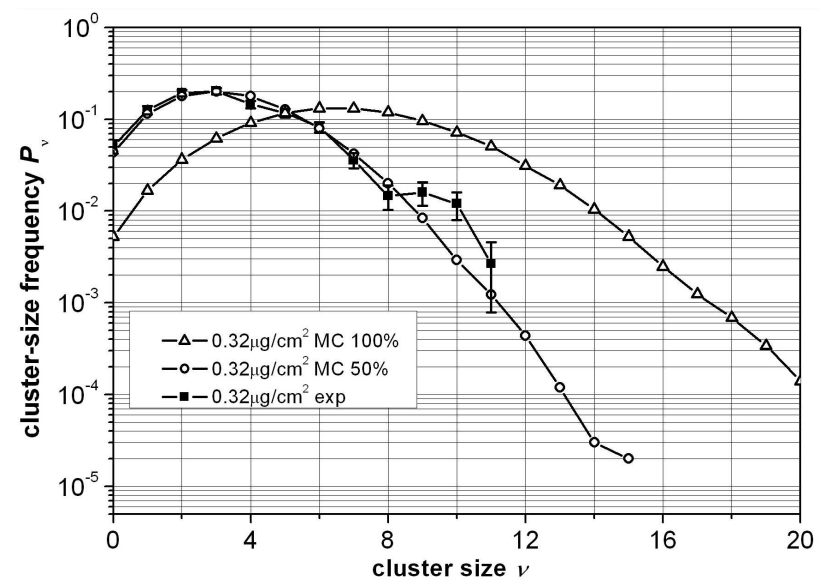

Fig. 4. Cluster size ionization spectra, $P_{v}$, for $76 \mathrm{MeV}$ carbon ions crossing centrally $0.32 \mu \mathrm{g} / \mathrm{cm}^{2}$ diameter cylindrical sensitive volume. Experimental results and Monte Carlo fits. Explanation of the plots seen at left lower corner.

Using the scaling procedure presented by Grosswendt [15], our SNS with $\mathrm{D} \rho=0.32 \mu \mathrm{g} / \mathrm{cm}^{2}$ in nitrogen corresponds to $2.2 \mathrm{~nm}$ in liquid water $\left(1 \mathrm{~g} / \mathrm{cm}^{3}\right)$. Based on ICSD spectra the new descriptors of the radiation damage for DNA scale are derived, namely the mean cluster size, $M_{1}$, created at $2.2 \mathrm{~nm}$ track segment equal to around 7 and cumulative probability, $F_{k}$, for multiple breaks equal to about 1 . The results are seen in Table.

TABLE

Results of the derived values (from ICSD spectrum seen in Fig. 4) of mean cluster size $M_{1}$ and cumulative probability $F_{2}$ for $76 \mathrm{MeV}$ carbon ions crossing the $0.32 \mu \mathrm{g} / \mathrm{cm}^{2}$ diameter sensitive volume.

\begin{tabular}{c|c|c}
\hline \hline$E[\mathrm{MeV}]$ & $M_{1}$ & $F_{2}$ \\
\hline 76 & 7.0 & 0.98
\end{tabular}

\section{Discussion and conclusion}

High LET radiation induces complex DNA damage, a class of DNA lesions that includes two or more individual types of lesions within one or two helical turns of the 
DNA [16]. DNA sensing proteins localize to the sites of DNA DSB resulting in the formation of microscopically visible nuclear domains referred to as radiation-induced foci in the $\gamma-\mathrm{H} 2 \mathrm{AX}$ assay [17].

Carbon ions penetrating through a nanometric track segment about $2.2 \mathrm{~nm}$ at unit density (comparable with DNA diameter) produce around 7 ionizations within the single carbon ion track [5]. The probability of the creation of the complex DSB damage is described by the cumulative probability $F_{2}$ for creation two or more lessions and is close to 1 as seen from Table. In general, this amount of ionizations is enough to produce complex damage in DNA strain breaks if it hits directly the DNA helix. Therefore, the $\gamma-\mathrm{H} 2 \mathrm{AX}$ assay shows many foci, statistically distributed within one track as seen in Fig. 3, creating very good visible patterns in the nucleus of the cell, reflecting ion traces. Unfortunately, through the nonspecificity of the method, the number of DSBs formed in the cell nucleus cannot be accurately determined and therefore the $\gamma-\mathrm{H} 2 \mathrm{AX}$ assay is not an ideal quantitative indicator of dose.

\section{Acknowledgments}

The authors would like to thank Dr. N. Keeley for a critical reading of the manuscript and for constructive suggestions.

A. Bantsar and S. Pszona contribution to this work are partially financed by BioQuaRT Euramet project.

\section{References}

[1] M. Durante, Br. J. Radiol. 87, 20130626 (2014).

[2] M.A. Tabocchini, A. Campa, V. Dini, Health Phys. 103, 547 (2012).

[3] B. Grosswendt, S. Pszona, A. Bantsar, Radiat. Prot. Dosim. 126, 432 (2007).
[4] J. Czub, D. Banaś, J. Braziewicz, J. Choiński, M. Jaskóła, A. Korman, Z. Szefliński, A. Wójcik, Radiat. Prot. Dosim. 122, 207 (2006).

[5] A. Bantsar, M. Pietrzak, M. Jaskóła, A. Korman, S. Pszona, Z. Szefliński, Rep. Prac. Oncol. Radiother. 19, 42 (2014).

[6] A. Bantsar, Ph.D. Thesis, e-Print: arXiv:1207.6893, The Andrzej Sołtan Institute for Nuclear Studies, Warszawa 2011.

[7] J. Czub, D. Banaś, A. Błaszczyk, J. Braziewicz, I. Buraczewska, J. Choiński, U. Górak, M. Jaskóła, A. Korman, A. Lankoff, H. Lisowska, A. Łukaszek, Z. Szefliński, A. Wójcik, Int. J. Radiat. Biol. 84, 821 (2008).

[8] M. Durante, J.S. Bedford, D.J. Chen, S. Conrad, M.N. Cornforth, A.T. Natarajan, D.C. van Gent, G. Obe, Mutat. Res. 756, 5 (2013).

[9] W.P. Roos, B. Kaina, Cancer Lett. 332, 237 (2013).

[10] K. Rothkamm, S. Horn, Ann. Ist. Super. Sanit. 45, 265 (2009).

[11] S.V. Costes, I. Chiolo, J.M. Pluth, M.H. BarcellosHoff, B. Jakob, Mutat. Res.-Rev. Mutat. 704, 78 (2010).

[12] M. Shrivastav, L.P. De Haro, J.A. Nickoloff, Cell Res. 18, 134 (2008).

[13] T. Nikolova, M. Dvorak, F. Jung, I. Adam, E. Krämer, A. Gerhold-Ay, B. Kaina, Toxicol. Sci. 140, 103 (2014).

[14] F. Bouquet, C. Muller, B. Salles, Cell Cycle 5, 1116 (2006).

[15] B. Grosswendt, Radiat. Prot. Dosim. 122, 404 (2006).

[16] D.J. Brenner, J.F. Ward, Int. J. Radiat. Biol. 61 , 737 (1992).

[17] T.E. Schmid, O. Zlobinskaya, G. Multhoff, Curr. Genomics 13, 418 (2012). 